\title{
Numerical Simulation of Closing Process of Shuttle Check Valve
}

\author{
Xiang Kefeng ${ }^{*}, 1,2$, Yao Jin $^{1}$ and Zeng Xiangwei ${ }^{2}$
}

\author{
${ }^{1}$ Sichuan University of Manufacturing Science and Engineering, Chengdu, Sichuan, 610041, China \\ ${ }^{2}$ Southwest University of Science and Technology of Manufacturing Science and Engineering, Mianyang, Sichuan, \\ 621010, China
}

\begin{abstract}
For studying the kinetic characteristics of passive shuttle check valve at the moment that it closes its valve core on the condition of high pressure differences, this paper first constructs the equation of motion of the valve core and twodimensional geometrical model by using computation fluid dynamics method, then with the help of the calculation method and physical model provided by Fluent software, employing the dynamic mesh and user-defined functions, simulates the unsteady flow in the valve core's closing process under the different pressure fluctuations, and obtains the stress nephogram, the velocity vector diagram, the speed curve, and pressure curve of the interior flow field of the valve. The results show that the bigger the value of valve fluctuation is, the larger the fluid pressure that the valve core is subjected to, the openness of the core and the speed fluctuation of the core are, and that in the case of sudden decompression at the entrance, the core can be shut down quickly with the steady speed field and pressure field. The simulation results are conducive to the design and optimization of structure parameter of shuttle check valve.
\end{abstract}

Keywords: Closing process, numerical simulation, shuttle check valve.

\section{INTRODUCTION}

The shuttle check valve invented by Zeng Xiangwei can automatically open and close itself under the control of the energy of the pipeline system, needing no extra power or control. The shuttle check valve can be closed safely and quickly in a relatively small back pressure due to its reasonable structure [1], and it now has been used in tens of large chemical plants and oil plants in China. In recent years, many researchers have been studying the shuttle check valve [2-6]. Li Liangchao [7] finished the numerical simulation of the opening process of shuttle valves; Chen Kun [8] analyzed the characteristic of the water attack in the shuttle valve pipe system; RenYuxin [9] simulated the noise-reduction feature of the shuttle valve. Yet few studies on the valve score's activity during the closing process of shuttle valve under the fluctuant pressure have come out. Simulation of the valve has been developed from the steady simulation at a certain opening degree to the non-steady simulation under a certain motion law. Therefore, it is necessary to study the impact of the pressure differences between the inlet and the outlet upon the closing process of the valve. The paper simulates the motion of the valve clack during the valve closing; provides the reference to the design of the structure parameter of the shuttle check valve and optimization.

\section{STRUCTURE OF SHUTTLE CHECK VALVE}

The structure of shuttle check valve is shown in Fig. (1). The outside is the valve body and the inside is the spool, and

*Address correspondence to this author at the School of Manufacturing Science and Engineering, Sichuan University, Sichuan, 621010, P.R. China; Tel: 13990193382; E-mail: xkf523@163.com a spring is placed in the rear of valve spool for pretightening. When medium flows into the left side of the picture, and the fluid's pressure on the spool is larger than the pressure of the spring and fluid from the right on the spool, the valve will move towards the right, and consequently valve is opened and the fluid will flow from the left to the right. When the pressure of the fluid from the left is less than the pressure of the fluid from the right and the spring's acting force, the spool will move to the left and consequently the valve is closed and backflow is prevented. Because the closing results from the spring's force and the pressure differences of both sides, therefore, the shuttle check valve can act as non-return function even in the case of the lowest backpressure [10]. Functionally speaking, the shuttle check valve can function as opening and closing, throttling, damping, and amplify regulator and controller.

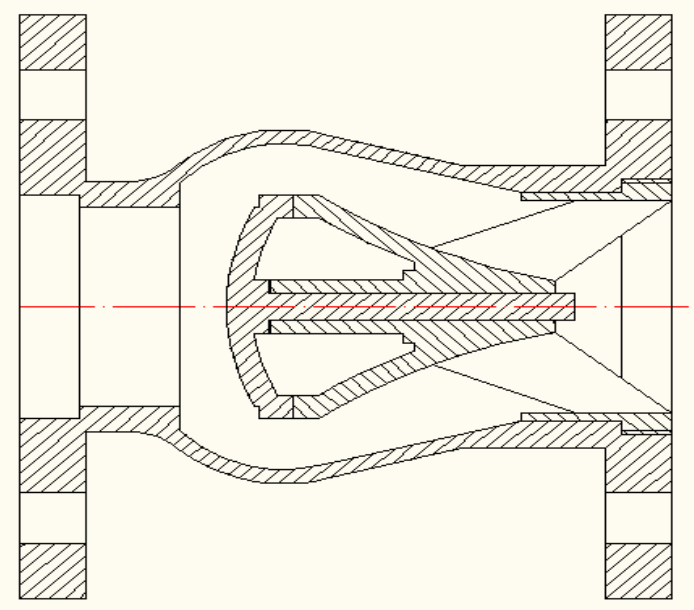

Fig. (1). Structure of shuttle check valve. 


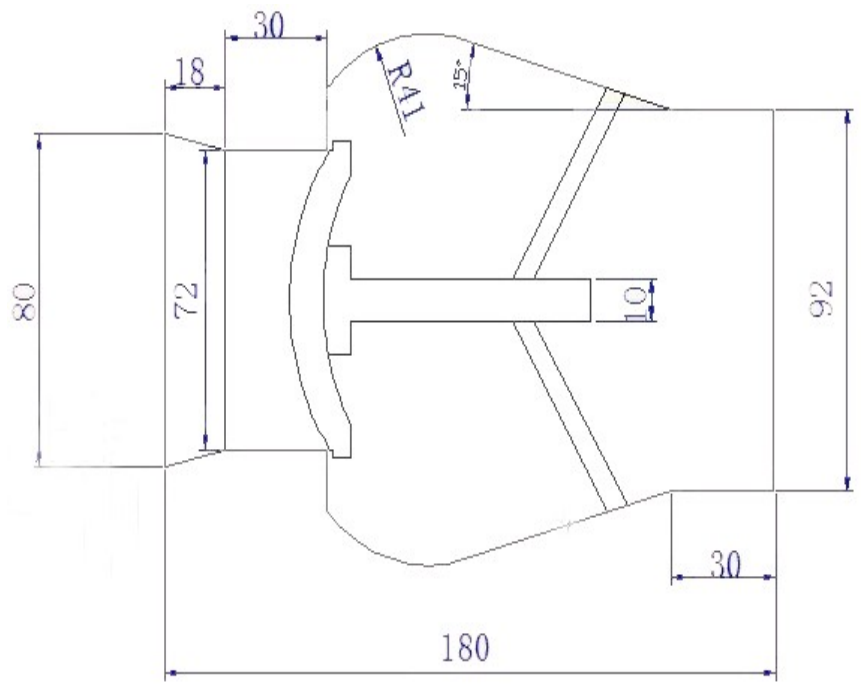

Fig. (2). Model diagram of shuttle valve.

The model of shuttle check valve and size are as Fig. (2) shows, the left side is the entry of the fluid (in the model the inlet diameter is $80 \mathrm{~mm}$ ), the right side is the outlet of the valve (the outlet diameter is $92 \mathrm{~mm}$ ), the total length of the valve is $180 \mathrm{~mm}$.

\section{BOUNDARY CONDITION AND PARAMETERS}

The mimetic shuttle check valve is the simplified version of the real one. The structure is completely axisymmetric, so it is feasible to build only half of it when building the geometric model with Gambit, and shape the whole part by symmetrical method. The check valve controls the final route through the valve seat; meanwhile the valve seat can direct the valve spool to move along horizontal axis. The simplified shuttle valve is as Fig. (3), considering the spool may appear cavitations if part of the inside have negative pressure during the moving process, the medium of the simulation is often the water of normal temperature or water vapor. The numerical simulation of the closing process of the shuttle valve is done under the condition of the pressure differences between the outlet and inlet being $0.4 \mathrm{MPa}$, and the elastic coefficient $6000 \mathrm{~N} / \mathrm{m}$.

Because it is dynamic simulation, triangular unstructured grid is chosen. The grids on the spool's left side and the tangent part of the valve body are intensified while the ones in other parts are loose. The total number of the grids is 39119.

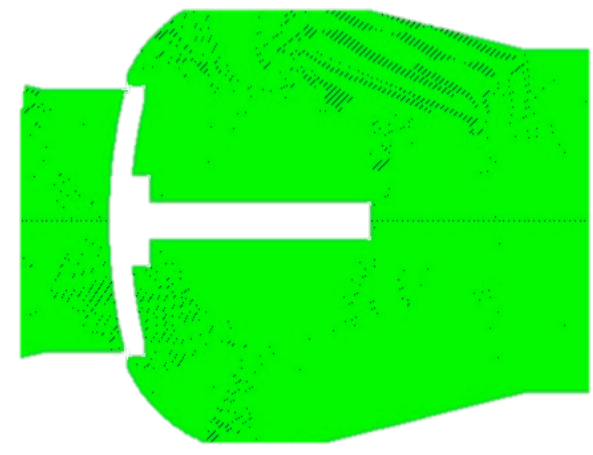

Fig. (3). Mesh map of shuttle valve model.
The dynamic simulation of the closing process of shuttle valve is the dynamic simulation of the valve spool's whole process from closing to complete opening. In the simulation, standard $\mathrm{k}-\varepsilon$ model is used as turbulence model, and SIMPLE arithmetic is used in the coupling of speed and pressure.

\section{CALCULATION MODEL AND PARTITION OF DYNAMIC MESH}

Dynamic mesh is controlled by the motion equation of the spool shown as formula 1 which is controlled by Newton's second law.

$m \frac{d^{2} x}{d^{2} t}=\int_{A} p_{i} d_{A}-F_{t}-f$

In the formula $1, \mathrm{~m}$ is the mass of the valve spool; $\mathrm{Ft}$ is the spring force $F_{t}=k \Delta x ; \mathrm{f}$ is the friction force, which can be ignored; $p_{i}$ is the fluid pressure received by the valve spool in the $\mathrm{x}$ direction; $\mathrm{A}$ is the area of the valve spool.

With dynamic mesh technique, flux $\Phi$, within the arbitrary control volume $\mathrm{V}$, its boundary conditions are dynamic, whose conservation equation general formula is:

$\frac{d}{d t} \int_{v} \rho \Phi d V+\int_{\partial v} \rho \Phi\left(u-u_{s}\right) d \cdot A=\int_{\partial v} \Gamma \nabla \Phi \cdot d A+\int_{v} S_{\Phi} d V$

In the formula $2, \rho$ is the fluid density, $u$ is the fluid velocity vector: $u_{s}$ is grid's strain rate of the dynamic mesh, $\Gamma$ is the diffusion coefficient, $S_{\Phi}$ is the source item of flux $\Phi ; \partial V$ is the boundary. The first item can be obtained by formula 3 .

$\frac{d}{d_{t}} \int_{V} \rho \Phi d V=\frac{(\rho \Phi d V)^{n+1}-(\rho \Phi d V)^{n}}{\Delta t}$

In the formula, $n$ and $n+1$ represents the present time and the next time point. $V^{n+1}$ can be obtained by formula 4 :

$V^{n+1}=V^{n}+\frac{d V}{d t} \Delta t$

In the formula, $\frac{d V}{d t}$ is time derivative of control unit. $\frac{d V}{d t}$ can be obtained by formula 5 :

$\frac{d V}{d t}=\int_{\partial V} u_{s} \bullet d A=\sum_{j}^{n f} u_{s j} A_{j}$

In the formula, $n f$ is the area of control body, $A_{j}$ is the superficial area of $\mathrm{j}$ side, $u_{s j} \bullet A_{j}$ is the dot of the control volume, it can be counted by formula 6 .

$u_{s j} \bullet A_{j}=\frac{\delta V j}{\Delta t}$ 
In the formula, $\delta V j$ is the change of the volume caused by the swelling of side during the overall time step $\Delta t$.

\section{NUMERICAL SIMULATIONS AND ANALYSIS}

In the shuttle valve's working process, the pressure of the entry always fluctuates and even disappears suddenly for the sake of production or accidents. Therefore, it is necessary for guarantying the safety and stability of the shuttle to analyze in detail the shuttle valve's working process. Here, the pressure between the inlet and outlet is $4000 \mathrm{~Pa}$; the elastic coefficient is $6000 \mathrm{~N} / \mathrm{m}$. The dynamic simulation of the closing process of the shuttle valve is done.

\subsection{Speed Vector Diagram and Pressure Field Diagram}

With the pressure difference of the outlet and inlet being $0 \mathrm{~Pa}$, the valve spool will move towards the inlet under the force of the spring, and therefore the valve is closed. Therefore, in simulating the shuttle valve's closing process, when it open to a steady moment, (here $0.5 \mathrm{~s}$ ), change the pressure difference of the outlet and inlet as $0 \mathrm{~Pa}$, and then make a dynamic simulation of it.

Comparing Fig. (4) with Fig. (5), we can see that the speed in the inlet keeps decreasing until it reaches the speed of $0 \mathrm{~m} / \mathrm{s}$ during the closing process. In the closing processing, the maximum bands velocity along the valve wall is decreasing and the speed value is decreasing too. On the contrary, the speed around the valve rod keeps increasing, which results from the backflow of the fluid on the right. During the closing, the right side of the valve spool will appear the blank area of flow rate, whose area keeps increasing as well, arising from the impact of the backflow.

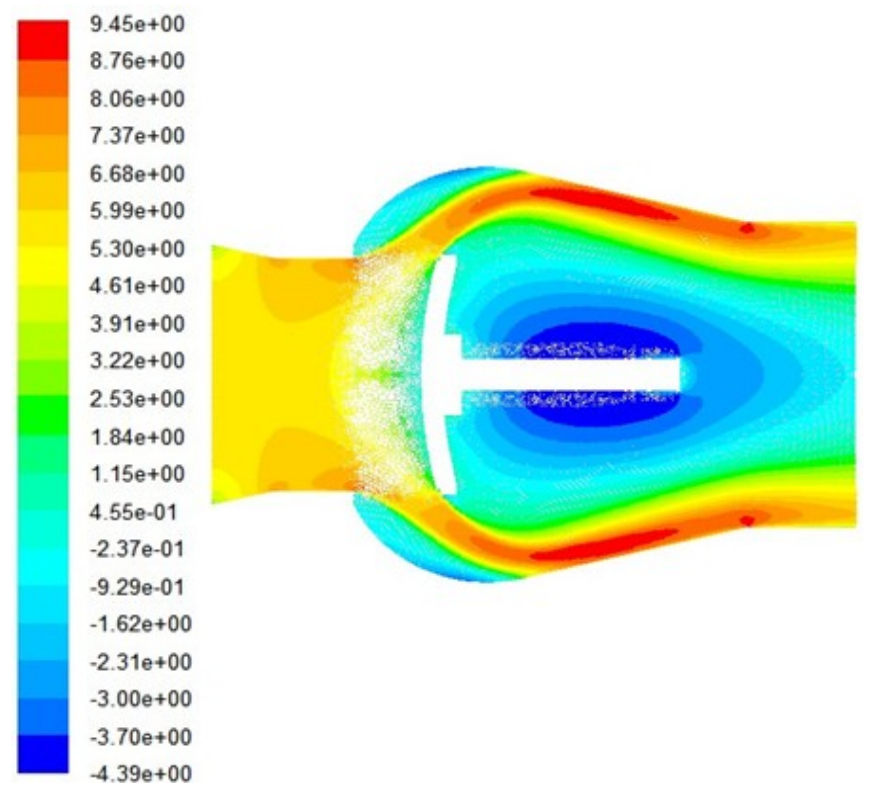

Fig. (4). Velocity vector in shuttle valve in the stable.

From Figs. $(6,7)$, it is easy to find then the pressure in the inlet disappear suddenly, the pressure distribution of the left side of the valve spool at the $0.002 \mathrm{~s}$ is basically returning to the previous state before decompression. And then the pressure distribution of the left side of the valve increases first and decrease until it comes to close. In the short period of decompression, the pressure distribution of the right side increases greatly, because the pressure around the valve rod increases greatly. At the $0.002 \mathrm{~s}$ the pressure distribution of the right side of the valve spool can resume to the previous state before decompression, and the pressure value of the outlet become larger. After $0.002 \mathrm{~s}$, the pressure values of both sides of the valve rod are relatively small; the pressure value of the area between the end of the valve rod and the outlet is relatively large.

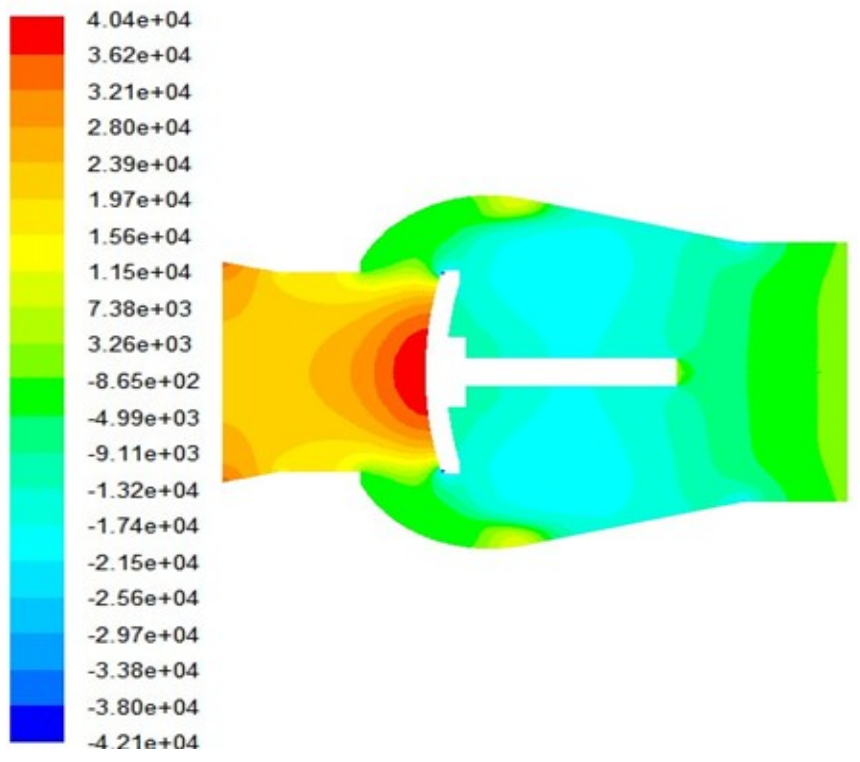

Fig. (5). Pressure contour in shuttle check valve in the stable.

\subsection{The Numerical Analysis of Simulation of the Closing Process}

The curve of valve spool by fluid pressure in shuttle check valve during closing process is as Fig. (8). At the 0.5 $\mathrm{s}$, the pressure in the entry suddenly decreases to $0 \mathrm{~Pa}$. From Fig. (8), we can see the pressure of the valve spool $0.001 \mathrm{~s}$ after the decompression abruptly decreases to $-726.42 \mathrm{~N}$ (This results from the sudden disappearing of the pressure of the inlet); at $0.002 \mathrm{~s}$ after decompression it returns to 123.66 $\mathrm{N}$; and at later phases the fluid pressure of the valve spool decreases steadily to $0 \mathrm{~N}$.

The Shuttle valve spool's displacement cure during the closing process is as Fig. (9). At the moment $0.5 \mathrm{~s}$, the pressure of the inlet disappears suddenly and the spool move towards the inlet with the speed increasing and then decreasing until its total close. Make a numerical simulation of the closing process, on the moment of $0.38 \mathrm{~s}$, the opening degree of the valve spool appear negative value, because there is $1 \mathrm{~mm}$ space between the spool and the body for the sake of division of grids in the modeling of the shuttle valve.

The curve of velocity vectors of valve spool in the shuttle check valve during the closing process is as Fig. (10) shows. From the curve we can see the valve spool's speed are negative during the closing process of the shuttle valve. As time goes on, the speed of valve spool keeps increasing; after the maximum absolute speed $-0.99 \mathrm{~m} / \mathrm{s}$, the speed of valve spool decreases. 
$\mathrm{t}=\mathbf{0 . 0 0 1 \mathrm { s }}$
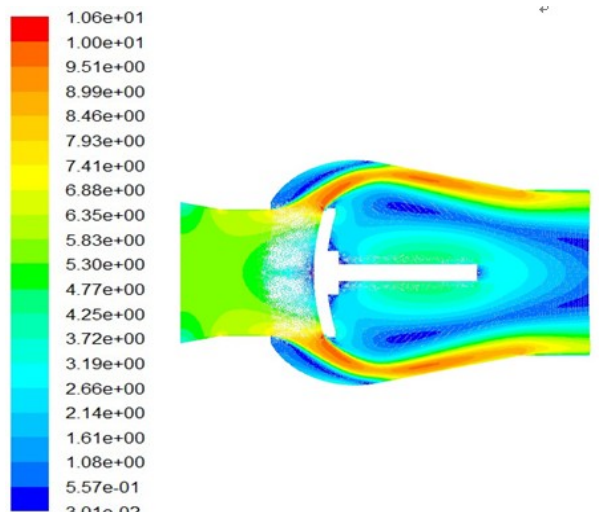

$\mathrm{t}=\mathbf{0 . 0 0 2} \mathrm{s}$

$1.03 \mathrm{e}+0$
$9.75 \mathrm{e}+00$

$924 \mathrm{e}+00$

$8.73 \mathrm{e}+00$

$8.21 \mathrm{e}+00$

$7.70 \mathrm{e}+00$

$7.19 \mathrm{e}+00$

$6.16 \mathrm{e}+00$

$5.65 \mathrm{e}+00$

$5.14 \mathrm{e}+00$

$4.63 \mathrm{e}+00$

$4.12 \mathrm{e}+00$

$3.60 \mathrm{e}+00$

$3.09 \mathrm{e}+00$

$2.58 \mathrm{e}+00$

$2.07 \mathrm{e}+00$

$1.55 \mathrm{e}+00$

$1.04 \mathrm{e}+00$

5. $29 \mathrm{e}-01$

1 64e-0?

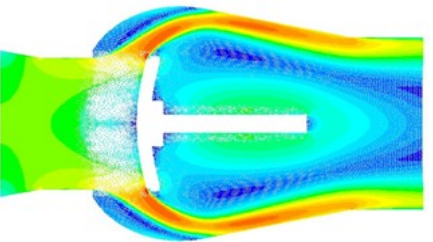

\section{$t=0.026 \mathrm{~s}$}

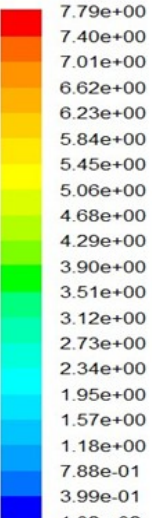

1.02e-02

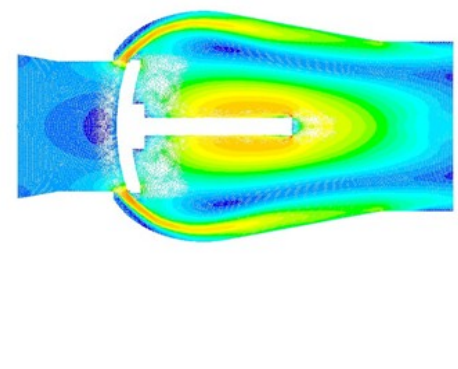

\section{$t=0.04 \mathrm{~s}$}

$5.05 \mathrm{e}+00$
$4.79 \mathrm{e}+00$
$4.52 \mathrm{e}+00$
$4.26 \mathrm{e}+00$
$3.99 \mathrm{e}+00$
$3.72 \mathrm{e}+00$
$3.46 \mathrm{e}+00$
$3.19 \mathrm{e}+00$
$2.93 \mathrm{e}+00$
$2.66 \mathrm{e}+00$
$2.40 \mathrm{e}+00$
$2.13 \mathrm{e}+00$
$1.87 \mathrm{e}+00$
$1.60 \mathrm{e}+00$
$1.33 \mathrm{e}+00$
$1.07 \mathrm{e}+00$
$8.04 \mathrm{e}-01$
$5.38 \mathrm{e}-01$
$2.73 \mathrm{e}-01$
$704 \mathrm{e}-03$

Fig. (6). Velocity vectors in shuttle check valve during closing process at different times. $\mathrm{t}=\mathbf{0 . 0 0 1 \mathrm { s }}$
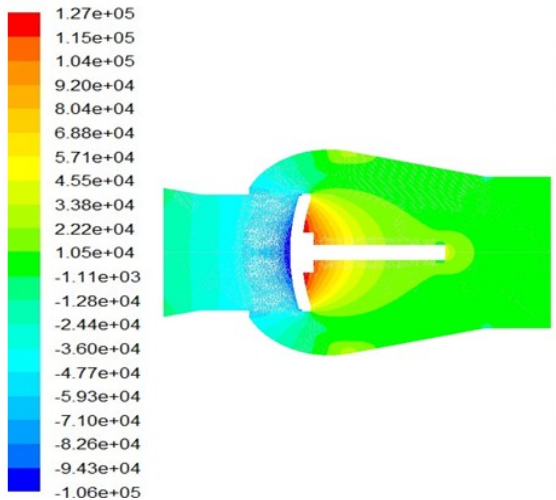

$\mathrm{t}=\mathbf{0 . 0 0 2 ~ s}$
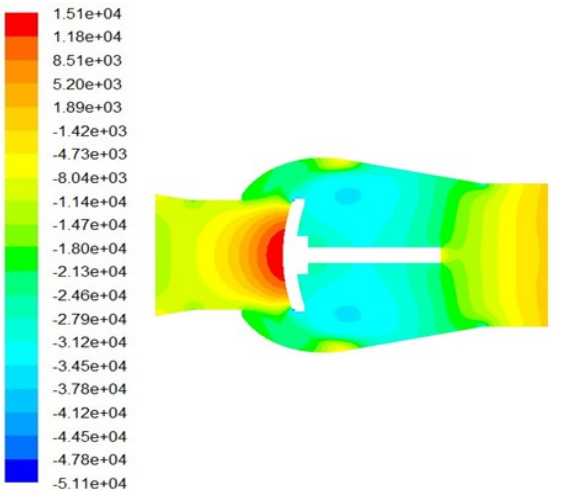

$45 \mathrm{e}+04$

$78 \mathrm{e}+04$

$45 \mathrm{e}+04$

$5.11 \mathrm{e}+04$

$\mathbf{t}=\mathbf{0 . 0 2 6} \mathrm{s}$

$4.28 \mathrm{e}+03$
$2.10 \mathrm{e}+03$

$2.10 \mathrm{e}+03$

$-6.88 \mathrm{e}+0$

$-2.24 \mathrm{e}+03$
$-4.41 \mathrm{e}+03$

$-4.41 \mathrm{e}+03$
$-6.58 \mathrm{e}+03$

$-8.76 \mathrm{e}+03$

$-1.09 \mathrm{e}+04$

$-1.31 \mathrm{e}+04$

$-1.53 e+04$

$-1.74 \mathrm{e}+04$

$-1.96 \mathrm{e}+04$

$2.18 \mathrm{e}+04$

$-2.40 \mathrm{e}+04$

$-2.61 e+04$

$-2.83 e+04$

$-3.05 e+04$

$-326 \mathrm{e}+04$

$-3.26 \mathrm{e}+04$

.

$3.70 \mathrm{e}+04$

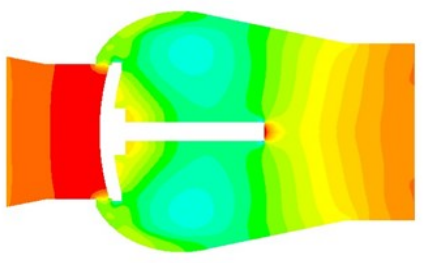

$\mathbf{t}=\mathbf{0 . 0 4} \mathrm{s}$

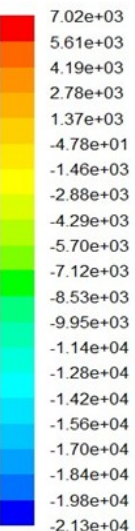

Fig. (7). Pressure contour in shuttle check valve during closing process at different times. 


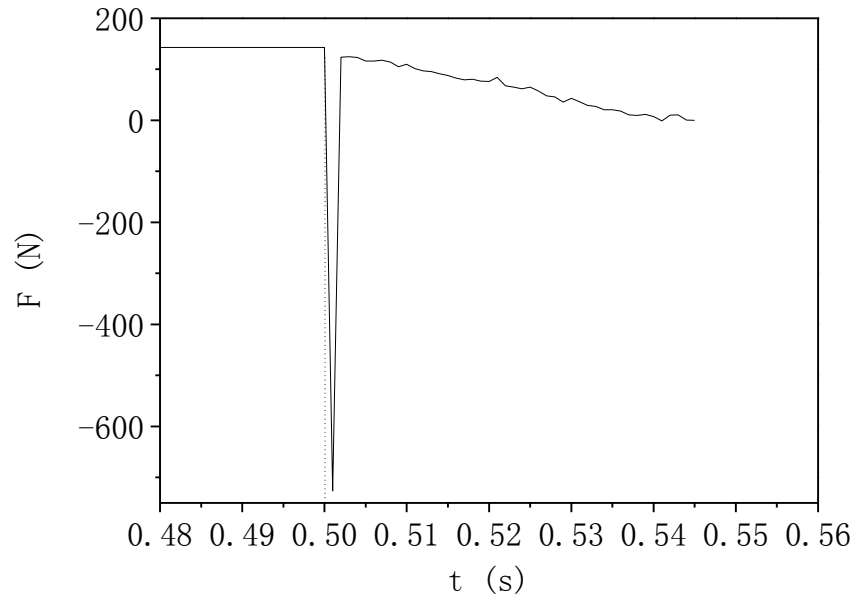

Fig. (8). The curve of valve spool by fluid pressure in shuttle check valve during closing processat.

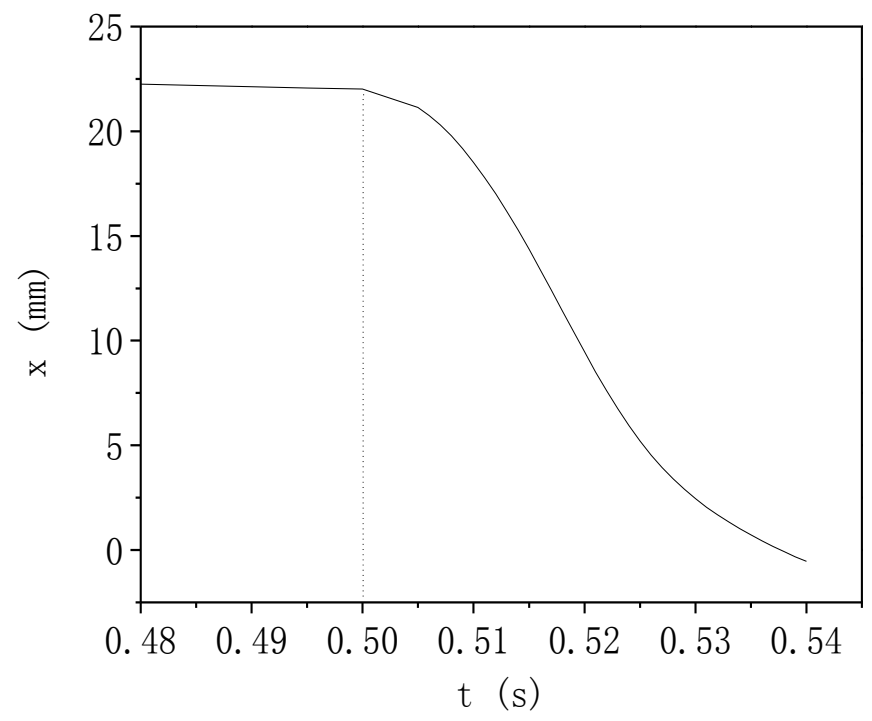

Fig. (9). The opening degree of valve spool in shuttle check valve during closing process.

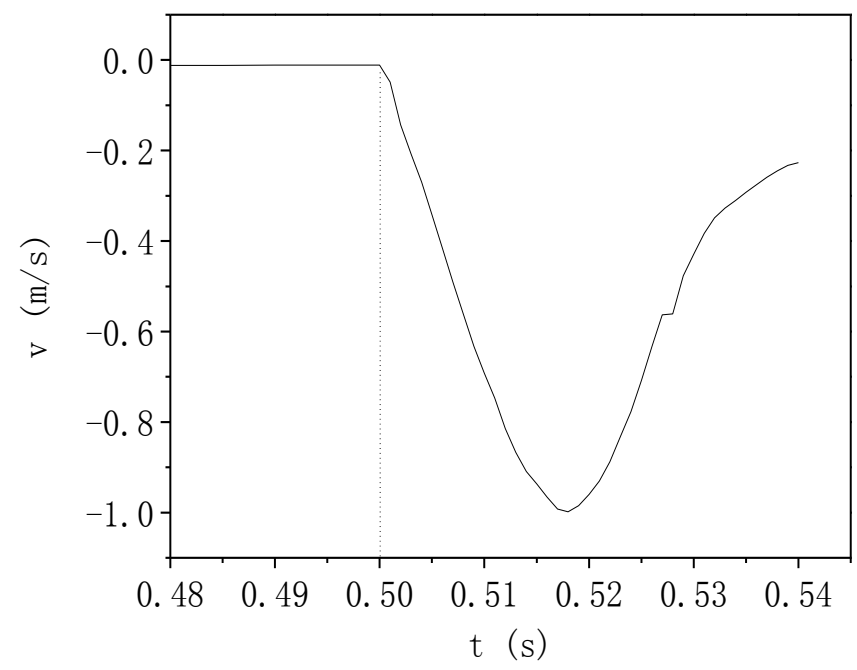

Fig. (10). The curve of velocity vectors of valve spool in shuttle check valve during closing process.
In the closing process, the speed of valve spool increases to the maximum, and then decreases until it's closed. Before the valve is closed, the spool is in a dynamic equilibrium system. Both the force acting on the spool and the distribution of velocity are even. The pressure of the flow field is stable so as to ensure the steady movement of the spool during the closing process, thus effectively avoiding the phenomena of the vibration, heating, cavitations, and guaranteeing the stability and safety of the shuttle valve in work.

\subsection{The Impact of Pressure Fluctuation on Dynamic Features}

To simulate the work of valve spool after its inlet pressure fluctuation becomes stable, change the pressure of the inlet and outlet at $0.48 \mathrm{~s}$, making the inlet pressure fluctuation (increase $5000 \mathrm{pa}, 1000$ pa separately). After 0.01 s fluctuation, the inlet pressure resumes to the original 40000 Pa. Figs. (11-13) are separately the pressure curve of the valve spool under different pressure fluctuation, its displacement curve and curve speed comparison diagrams.

The curve of valve spool by fluid pressure in different pressure fluctuation is as Fig. (11). When the pressure fluctuation is $5000 \mathrm{~Pa}$, the fluid pressure of the spool will resume its balance at $0.695 \mathrm{~s}$, and the fluid pressure of spool will resume to $142 \mathrm{~N}$ as well. When the pressure fluctuation is $10000 \mathrm{~Pa}$, the fluid pressure of the spool will resume its balance at $0.715 \mathrm{~s}$, and the fluid pressure of spool will resume to $142 \mathrm{~N}$ as well. From this we can see the more the pressure fluctuation is, the more the fluid pressure of the valve spool will be, and will achieve pressure stability in the long time after the pressure recovery. While after the balance, the pressure of the spool will all return to the value before the fluctuation.

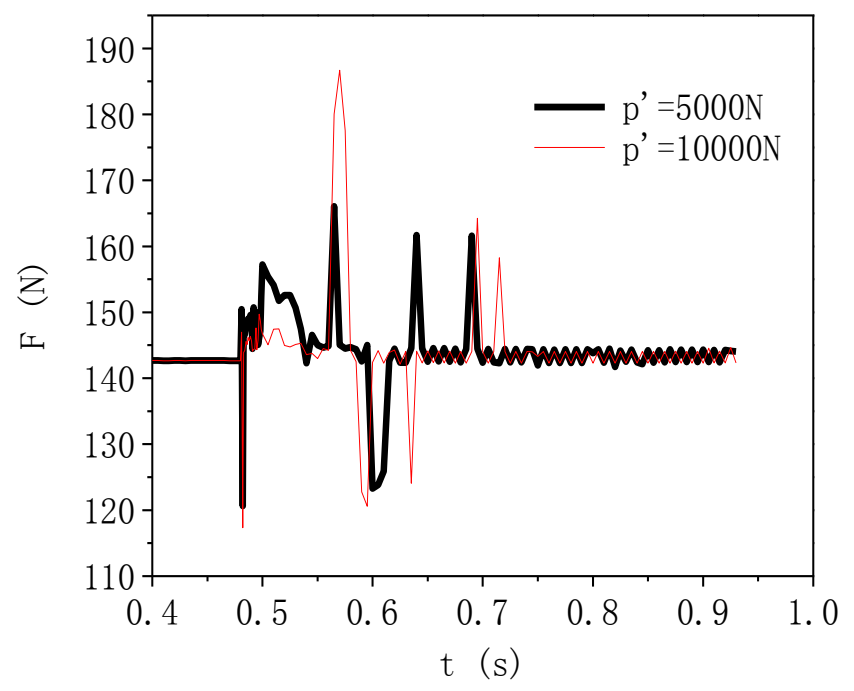

Fig. (11). The curve of valve spool by fluid pressure in different pressure fluctuation.

The displacement curve of valve spool in different pressure fluctuation is as Fig. (12). When the pressure is $5000 \mathrm{pa}$, the displacement of spool will change to $23.32 \mathrm{~mm}$; when the pressure is $10000 \mathrm{pa}$, spool's displacement will be 
24.55 at most. Here we can see, the bigger the pressure fluctuation is, and the bigger the valve spool's displacement will be. From the figure, we can also tell in the case of big pressure fluctuation, the openness of valve spool will be more quickly changed (including the speed of displacement reduction). But when the valve spool resume to its balance, the openness will resume to the value before the fluctuation.

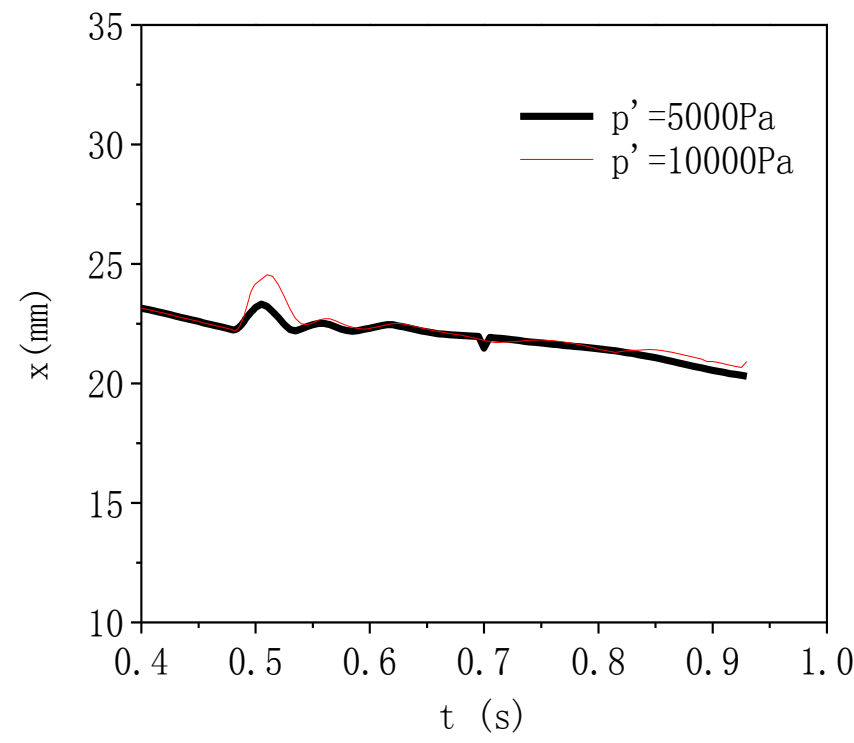

Fig. (12). The opening degree of valve spool in different pressure fluctuation.

The curve of velocity vectors of valve spool in different pressure fluctuation are as Fig. (13). When the pressure is $5000 \mathrm{pa}$, the biggest variation of the spool speed will be $0.073 \mathrm{~m} / \mathrm{s}$; when the pressure goes to $10000 \mathrm{~Pa}$, the biggest variation of the spool speed will be $0.175 \mathrm{~m} / \mathrm{s}$. Therefore, the bigger the pressure fluctuation, the bigger the range of the valve spool's speed will be. From this figure, the bigger the pressure fluctuation is, the more quickly the speed will vary.

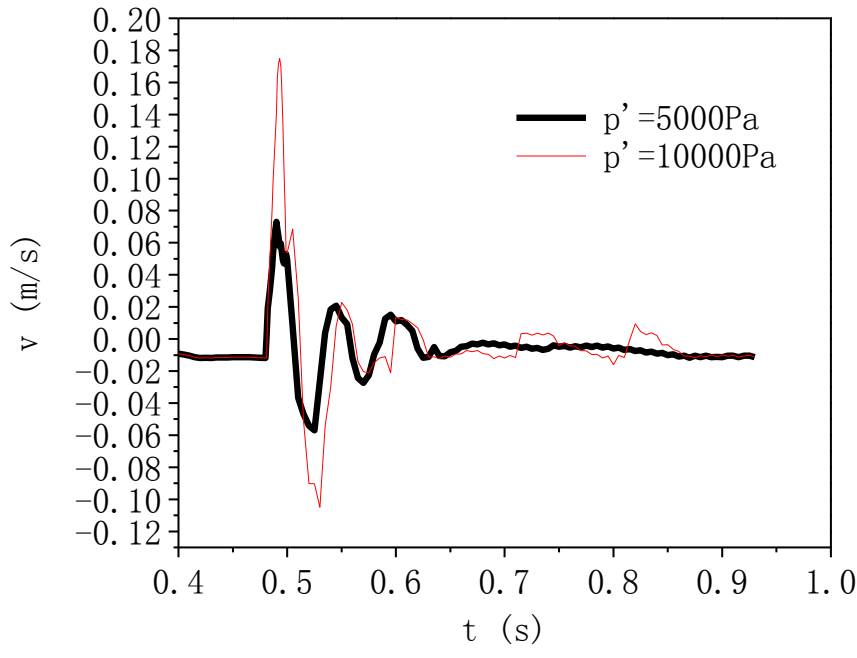

Fig. (13). The curve of velocity vectors of valve spool in different pressure fluctuation.

From all above, the bigger the pressure fluctuation is, the more the spool's pressure, displacement and speed will become. After its resumption of balance, the fluid pressure of the spool, the openness of the spool and the speed of the spool are the same with those before the pressure fluctuation.

\section{CONCLUSION}

The paper does a numerical simulation of the closing process of the shuttle valve spool when it meets pressure fluctuation and the decompression happens in the entrance of the shuttle valve. So conclusions are as follows:

1. In the closing process of the shuttle valve, the spool gets its force evenly, the velocity distribution are even, pressure of flow field is stable, ensuring the steady movement of shuttle valve spool in the closing process. Thus the phenomena of vibration, heating, cavitations in relation to valve spool can be effectively reduced, guaranteeing the stability and safety of shuttle valve in the pipeline transportation and improves the working life of the shuttle valve.

2. The bigger the pressure fluctuation is, the bigger the fluid pressure fluctuation of the valve spool will be, and reach the pressure steadiness again in a longer period when the pressure is resumed.

3. Relying on the pressure difference of directions forward and reverse, passive shuttle valve pushes shuttle spool to make a shift between opening and closing, safely realize its opening or closing. It can be proved with simulation that passive shuttle check valve receives little damage, the closing process is done quickly and stably with no shock, the spool can steadily match the valve seat, achieving good sealing. Passive shuttle check water-proof valve can reduce the water's pressure to the minimum, making the pressure pipeline system safe and reliable, and can serve as damping and denoising function.

\section{CONFLICT OF INTEREST}

The authors confirm that this article content has no conflict of interest.

\section{ACKNOWLEDGEMENTS}

Declared none.

\section{REFERENCES}

[1] X. Zeng, S. Huang, and S. Gao, "The application of the shuttle check valve in the fluid transport," Petroleum Refinery Engineering, vol. 30, no. 4, pp. 26-29, 2000. (in Chinese)

[2] K. Xiang, X. Zeng, and B. Xiang, "None dynamic protection of pipeline rupture," Oil-Gas Field Surface Engineering, vol. 31, no. 7, pp. 35-36, 2012. (in Chinese)

[3] K. Chen, X. Zeng, and D. Ren, "Research on the pressure conduit protection system by passive control," Manufacturing Automation, vol. 32, no. 1, pp. 118-121, 2010. (in Chinese)

[4] C. Mo, J. Zhu, and B. Xiang, "Structure optimization design of shuttle-type rupture protection device," Machinery Design and Manufacture, vol. 41, no. 8, pp. 219-221, 2013. (in Chinese)

[5] K. Chen, J. Huang and X. Qiu, "Shuttle-type intelligent pipeline protection device," Journal of Wuhan University of Technology, vol. 34, no. 1, pp. 88-92, 2010. (in Chinese)

[6] K. Chen, X. Zeng, and W. Chen, "The finite element analysis of Shuttle-type intelligent pipeline protection system," The Collection Pressure Pipeline Technology Research Progress, vol. 9, pp. 5155, 2010. (in Chinese) 
[7] L. Li, X. Zeng, and K. Xiang, "Numerical simulation of opening process of shuttle check valve," Journal of Drainage and Irrigation Machinery Engineering, vol. 30, no. 6, pp. 710-714, 2012. (in Chinese)

[8] K. Chen, X. Zeng, and W. Chen, "The water hammer analysis of pressure conduit burst protection system with intelligent control," Chinese Hydraulics and Pneumatics, vol. 1, pp. 45-47, 2010. (in Chinese)
[9] Y. Ren, J. Zhu, B. Xiang, andX. Zeng, "Research on the noise reduction performance of passive shuttle - type anti-water-attack check valve," Machine Tool and Hydraulics, vol. 39, no. 23, pp. 74-76, 2011. (in Chinese)

[10] Y. Cai, B. P. Xiang, and G. D Cheng, "Research of fluid-structure interaction performance of shuttle check valve," Coal Mine Machinery, vol. 33, no. 2, pp. 70-73, 2012. (in Chinese)

(C) Kefeng et al.; Licensee Bentham Open.

This is an open access article licensed under the terms of the Creative Commons Attribution Non-Commercial License (http://creativecommons.org/licenses/by-nc/3.0/) which permits unrestricted, non-commercial use, distribution and reproduction in any medium, provided the work is properly cited. 